
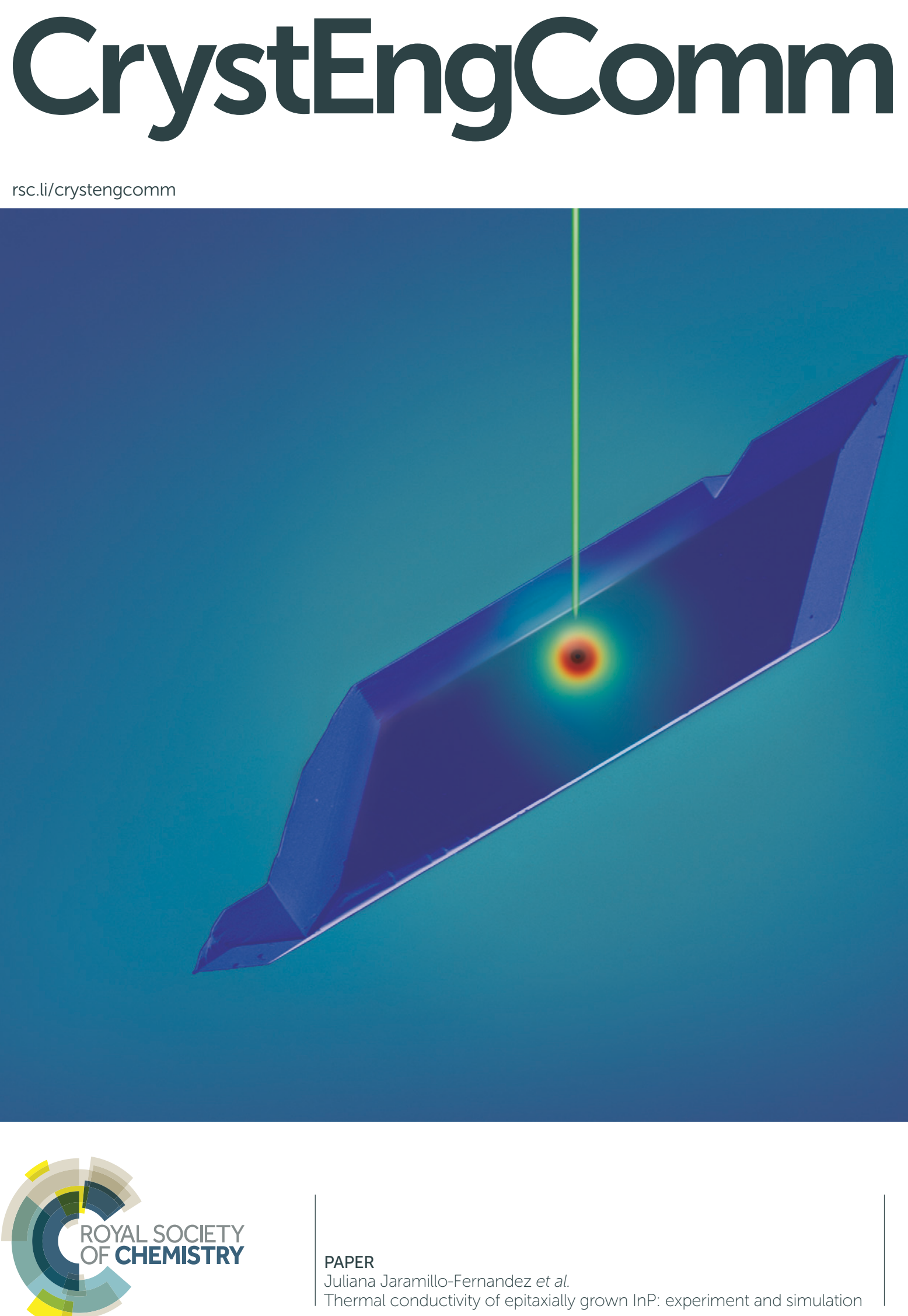


\title{
CrystEngComm
}

Check for updates

Cite this: CrystEngComm, 2017, 19, 1879

Received 27th December 2016 Accepted 10th February 2017

DOI: $10.1039 / c 6 c e 02642 g$

rsc.li/crystengcomm

\section{Thermal conductivity of epitaxially grown InP: experiment and simulation $\uparrow$}

\author{
Juliana Jaramillo-Fernandez, ${ }^{* a}$ Emigdio Chavez-Angel, ${ }^{\mathrm{b}}$ Reza Sanatinia, $\$^{\mathrm{a}}$ \\ Himanshu Kataria, $\S^{a}$ Srinivasan Anand, ${ }^{a}$ \\ Sebastian Lourdudoss ${ }^{a}$ and Clivia M. Sotomayor-Torres ${ }^{\text {abc }}$
}

\begin{abstract}
The integration of III-V optoelectronic devices on silicon is confronted with the challenge of heat dissipation for reliable and stable operation. A thorough understanding and characterization of thermal transport is paramount for improved designs of, for example, viable III-V light sources on silicon. In this work, the thermal conductivity of heteroepitaxial laterally overgrown InP layers on silicon is experimentally investigated using microRaman thermometry. By examining InP mesa-like structures grown from trenches defined by a $\mathrm{SiO}_{2}$ mask, we found that the thermal conductivity decreases by about one third, compared to the bulk thermal conductivity of InP, with decreasing width from 400 to $250 \mathrm{~nm}$. The high thermal conductivity of InP grown from $400 \mathrm{~nm}$ trenches was attributed to the lower defect density as the InP microcrystal becomes thicker. In this case, the thermal transport is dominated by phonon-phonon interactions as in a low defect-density monocrystalline bulk material, whereas for thinner InP layers grown from narrower trenches, the heat transfer is dominated by phonon scattering at the extended defects and $\ln \mathrm{P} /$ $\mathrm{SiO}_{2}$ interface. In addition to the nominally undoped sample, sulfur-doped $\left(1 \times 10^{18} \mathrm{~cm}^{-3}\right) \operatorname{InP}$ grown on $\mathrm{Si}$ was also studied. For the narrower doped InP microcrystals, the thermal conductivity decreased by a factor of two compared to the bulk value. Sources of errors in the thermal conductivity measurements are discussed. The experimental temperature rise was successfully simulated by the heat diffusion equation using the FEM.
\end{abstract}

\section{Introduction}

Remarkable advances achieved in the field of silicon photonics in the past decade have paved the way for an integrated optoelectronic platform based on silicon. For example, improved manufacturing paths have enabled the fabrication of low-loss passive devices such as filters, waveguides, beam splitters and combiners, which are now standard available devices. ${ }^{1-4}$ However, the development of active devices and, in particular, the integration of efficient light sources remain an open engineering challenge as far as heat dissipation is concerned. The successful fabrication of such integrated de-

\footnotetext{
${ }^{a}$ Department of Material and Nano Physics, Royal Institute of Technology KTH, Kista, SE-16440, Sweden. E-mail: jif@kth.se

${ }^{b}$ Catalan Institute of Nanoscience and Nanotechnology (ICN2), CSIC and The Barcelona Institute of Science and Technology, Campus UAB, Bellaterra, 08193 Barcelona, Spain

${ }^{c}$ ICREA, Passeig Lluis Companys 23, 08010 Barcelona, Spain

$\dagger$ Electronic supplementary information (ESI) available. See DOI: 10.1039/ c6ce $02642 \mathrm{~g}$

¥ Present address: John A. Paulson School of Engineering and Applied Sciences, Harvard University, Cambridge, Massachusetts 02138, USA.

$\S$ Present address: IRnova AB, Electrum 236, SE-164 40 Kista, Sweden.
}

vices holds the key to accomplishing the long-term dream of the photonics and electronics industries: a fully integrated III-V/Si platform.

Hybrid integration of III-V semiconductor optoelectronic devices by wafer bonding has been successfully employed on several devices, such as silicon-based modulators, photodetectors, amplifiers and coherent light sources. ${ }^{5-8}$ However, monolithic approaches are preferred for long-term, largescale and low-cost manufacturing of devices with enhanced yields. Yet, there are fundamental limitations to the direct growth of III-V active devices on $\mathrm{Si}$, which involve the strong lattice mismatch and large difference in thermal expansion coefficients between III-V semiconductors and silicon substrates. ${ }^{9-13}$ These factors result in high density threading dislocations and planar defects such as stacking faults, which ultimately lead to material degradation and shortened device lifetimes. The interaction of phonons with crystallographic defects, interfaces between components and layer boundaries results in higher temperatures in localized volumes, especially when the dimensions of the system are scaled down to the micro and nanometric scales. Thus, the increased scattering events of heat carriers significantly reduce the thermal conductivity and give rise to important thermal issues, 
primarily stability. ${ }^{14,15}$ The ability to effectively dissipate heat is therefore a crucial limiting factor in integrated photonic circuit design, due to the fact that device functionality and performance strongly depend on the temperature. In particular, for semiconductor coherent light sources, the yield usually decreases above room temperature while the threshold current increases rapidly with temperature.

To date, few studies have addressed thermal effects in integrated III-V on silicon device-like structures and most of them focus on hybrid (wafer-bonded) devices. Sysak et al. ${ }^{16}$ analyzed a hybrid laser integration platform from a thermal perspective using thermal impedance measurements and finite element simulations. They conclude that the dominant factor influencing the device thermal impedance is the buried oxide layer, which accounts for about $50 \%$ of the total thermal resistance. Lei and co-workers proposed an AlN cladding layer as a possible solution for heat spreading in a hybrid III-V laser on silicon, based on a multiphysics electrothermo-mechanical model. ${ }^{17,18}$ Recently, Lucci et al. ${ }^{19}$ theoretically investigated the thermal issues related to the monolithic or heterogeneous nature of a device. They suggest that monolithically integrated lasers dissipate heat towards the Si substrate more efficiently than their hybrid counterparts, arguing that misfit dislocations due to the III-V/Si heteroepitaxy do not impact significantly on the thermal properties of $\mathrm{GaSb} / \mathrm{AlSb} / \mathrm{Si}$ epitaxial layers in these lasers. Despite the increasing interest in thermal management in III-V semiconductor devices integrated on $\mathrm{Si}$, direct thermal conductivity measurements of the building block components of devicelike structures have not been reported so far and remain a challenging task. The main reason is the size of such structures, which is usually in the range of few micrometers. On this scale, traditional techniques used for bulk materials are no longer suitable to describe thermal transport. ${ }^{20}$ Accurate thermal conductivity determination is thus a pivotal step towards realistic thermal analysis in such structures. In this work, we report an experimental investigation of the thermal conductivity of heteroepitaxial laterally overgrown (ELOG) InP layers on silicon substrates. ELOG is one of the methods among several heteroepitaxial techniques used to grow III-V materials on $\mathrm{Si}^{21-23}$ It uses a dielectric mask $\left(\mathrm{SiO}_{2}\right.$ in our case) deposited on the buffer layer patterned with trenches through which the epilayers are grown. The buffer layer, called the seed layer, may contain a high dislocation density, on the order of $10^{9} \mathrm{~cm}^{-2}$. The dielectric mask between the trenches acts as a filter for crystallographic defects, therefore resulting in near defect-free mask-epilayer interfaces, and thus high crystalline quality InP on $\mathrm{Si}$. The dislocation density has been shown ${ }^{3}$ to be reduced to $6 \times 10^{6} \mathrm{~cm}^{-2}$. It can also offer the advantage of integrating the gain medium onto a $\mathrm{SiO}_{2} / \mathrm{Si} / \mathrm{SiO}_{2}$ waveguide for a monolithic evanescently coupled silicon laser. ${ }^{24}$ Micro-Raman spectroscopy is used to measure the thermal conductivity of the ELOG InP microcrystals, as a contactless and non-destructive optical technique particularly well suited for studying the thermal properties of micrometric samples with irregular geometries. Since build- ing blocks in functional devices are doped, we extend our study to a doped sample. A thorough analysis of errors in the measurement techniques is presented and the results are successfully simulated by the finite element method (FEM). Based on the experimental results, we analyze the interplay between the ELOG sample size and crystallographic defects on thermal transport for undoped and sulfur-doped InP microcrystals. The impact of our findings on coherent light source integration on $\mathrm{Si}$ is discussed.

\section{Experimental}

\subsection{Sample preparation}

High quality InP microcrystals were grown on Si by epitaxial lateral overgrowth. This approach for heterogeneous integration involves growing InP laterally from trenches in a mask deposited on a defective InP seed layer on a Si substrate. The patterned mask between the trenches acts as a filter for defects arising from differences in the lattice constants (8\%) and thermal expansion coefficients (of about 50\%) between InP and Si. In addition, if the trench width is half as much as the mask thickness, the so-called necking effect can filter the defects even in the grown region above the opening (Fig. 1). ${ }^{25}$

The specimens were prepared according to the procedure for heteroepitaxial growth of $\mathrm{InP}$ on $\mathrm{Si}$ reported elsewhere. $^{26,27}$ The samples consisted of high-quality epitaxial InP layers grown through trench openings patterned on a 600 nm thick $\mathrm{SiO}_{2}$ mask. The mask was deposited, prior to the epitaxial growth, on a highly defective InP seed layer grown on a $\mathrm{Si}$ substrate to accommodate the severely lattice mismatched materials. The width of these openings was varied from 250 to $400 \mathrm{~nm}$ to study its influence on crystal growth and the filtering of planar defects and dislocations. In order to have a better understanding of thermal conductivity in device-like structures, we investigated both undoped and sulfur-doped $\left(\sim 1 \times 10^{18} \mathrm{~cm}^{-3}\right)$ samples. Furthermore, the $\mathrm{SiO}_{2}$ layer was removed by wet etching from the first set of samples in order to evaluate its influence on thermal diffusion.

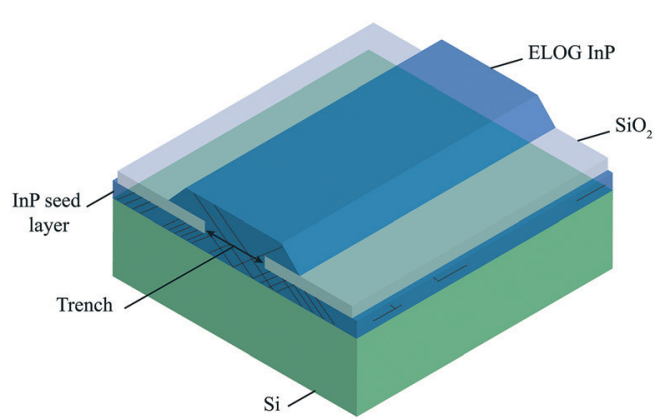

Fig. 1 Schematic illustration of the investigated samples obtained by heteroepitaxial growth of InP on Si using the ELOG technique. 


\subsection{Thermal conductivity measurements using the Raman shift method}

The thermal conductivity of InP microcrystals was measured by the Raman shift method. In standard Raman thermometry experiments, continuous visible light is partially absorbed by a semi-opaque sample, resulting in non-homogenous heating of the probed volume. The analyzed volume is given by $V=$ $4 \pi r^{2} \rho$, where $r$ is the radius of the Gaussian focused beam and $\rho$ is the penetration depth of light in the solid.

By gradually increasing the excitation power, the power absorbed at the surface increases, inducing a localized nonhomogeneous temperature rise. Simultaneously, the light that interacts with the studied sample undergoes Raman scattering, giving rise to a spectrum where the Raman frequencies of optical phonons are red-shifted due to the thermal expansion of the lattice. Owing to the variations in the equilibrium position of the atomic species, the anharmonicity of the bonds results in a variation of the interatomic forces and therefore in the shift of the phonon frequencies. ${ }^{28,29}$ The resulting temperature-dependent peak positions are then recorded as a function of the absorbed power and compared to a calibration curve that allows the estimation of the weighted average temperature rise in the probed volume. The calibration curve is obtained by externally heating the sample and registering the corresponding vibrational frequencies of the optical phonon modes. A schematic of the set-up used in the backscattering geometry to carry out the thermal conductivity measurements is shown in Fig. 2. An argon ion laser with a wavelength of $532 \mathrm{~nm}$ was simultaneously used as a heat source and a temperature measuring probe. The laser light was focused onto the sample surface by a $50 \times$ objective with a numerical aperture of NA $=0.55$. In Raman thermometry, the main sources of uncertainty on the measured thermal conductivity arise from the uncertainties on the laser beam radius and the power absorbed at the sample surface. These parameters were therefore carefully measured. The radius of the nearlyGaussian spot was obtained by recording the intensity of the first-order Si Raman peak while scanning the beam across the edge of a gold film deposited on a Si substrate (Fig. 3a).

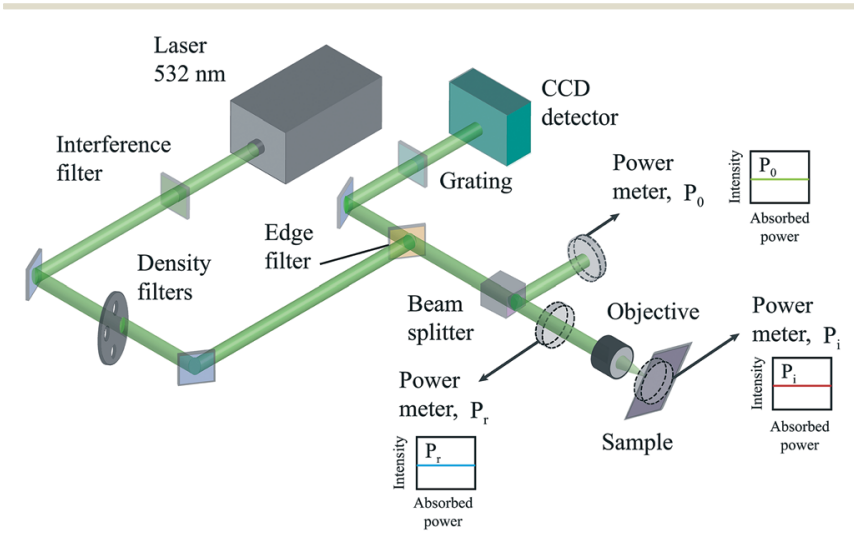

Fig. 2 Schematic of the Raman scattering measurement set-up with a built-in array of power meters used for thermal measurements and power absorption characterization.
The intensity of the Si peak was gradually attenuated until it completely disappeared when the spot fully reached the metallic area (Fig. 3b and c). A Gaussian function of the form $I \cdot \exp \left(-x^{2} /\right.$ $r^{2}$ ) was used to fit the slope of the intensity $I$ versus position $x$ plot (Fig. 3d), and thus a radius of $r=0.85 \mu \mathrm{m}$ was determined. From the absorption coefficient of InP at the excitation wavelength of $532 \mathrm{~nm}\left(\alpha=1.01 \times 10^{7} \mathrm{~m}^{-1}\right)$, the light penetration depth was estimated to be $\rho=98.7 \mathrm{~nm}$. Since Raman thermometry also requires the accurate determination of the absorbed power at the sample surface, we carried out in situ reflectance and absorbance measurements for various laser powers. The reflectance $R$, was obtained by measuring the incident $P_{\mathrm{i}}$ and reflected powers $P_{\mathrm{r}}$, using commercial power meters and considering that $R=P_{\mathrm{r}} / P_{\mathrm{i}}$. Given the considerable thickness of the InP microcrystals, which varied from 1.5 to $15 \mu \mathrm{m}$ depending on the trench width, the transmittance was neglected and the absorbance was estimated using the expression $A=1-R$. The inbuilt power meter set-up used for the absorbed power characterization is shown in Fig. 2 in dashed lines. The measured reflectance and absorbance of an InP microcrystal grown on Si through a $250 \mathrm{~nm}$ trench are shown in Fig. 4 as an example. The obtained experimental data at $532 \mathrm{~nm}$ do not vary within the measured range of absorbed power and match well with the reference values previously reported by Aspnes et al. ${ }^{30}$ Using the measured absorbance, we estimated that the power absorbed at the sample surface varied from about 3 to $15 \mathrm{~mW}$ when the incident laser power increased from about 6 to $22 \mathrm{~mW}$.

In general, the phonon Raman scattering spectra of zinc blende materials exhibit two peaks corresponding to the longitudinal optical (LO) and transversal optical (TO) phonons with the wave vector $\boldsymbol{k}=0$. In bulk InP, the first-order TO and LO Raman peaks have frequencies of $303.7 \mathrm{~cm}^{-1}$ and $345.0 \mathrm{~cm}^{-1}$, respectively. ${ }^{31}$ To correlate the phonon frequency shifts with the sample temperature rise, we characterized the dependence of
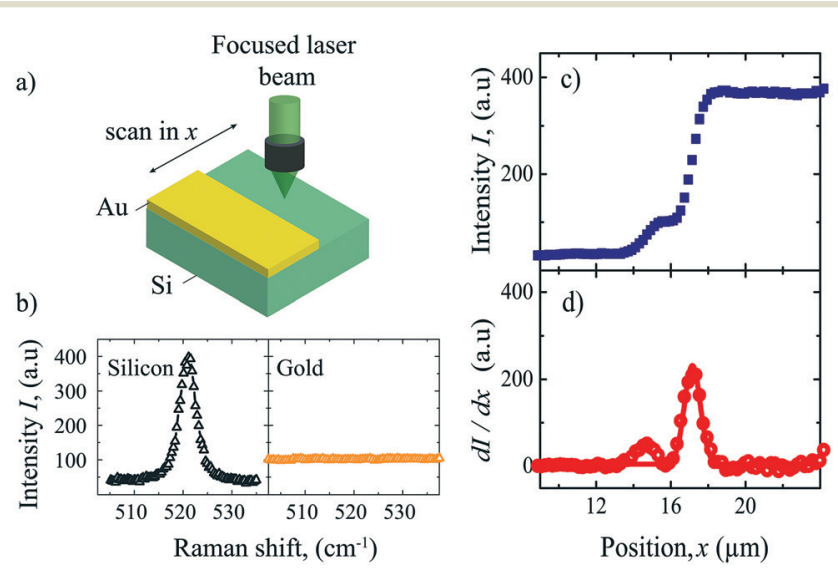

Fig. 3 a) Schematic illustration of the setup used for laser beam size measurements. b) Raman spectra of silicon and gold, depicted on the left and right panels, respectively. c) Intensity of the first-order Si Raman peak obtained by scanning the laser beam across the edge of a gold film deposited on a Si substrate. d) Derivative of the intensity with respect to the position, fitted by a Gaussian function (continuous line), from which the radius of the beam can be estimated. 


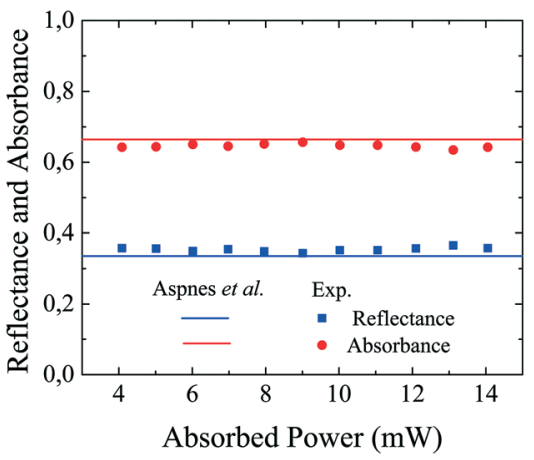

Fig. 4 Experimental reflectance and absorbance of an $\ln P$ microcrystal grown on $\mathrm{Si}$ in a $250 \mathrm{~nm}$ trench opening on the $\mathrm{SiO}_{2}$ mask. The continuous lines represent the data reported by Aspnes et al. ${ }^{30}$ for single crystalline $\mathrm{InP}$ at $532 \mathrm{~nm}$.

the LO Raman shift on the temperature. The sample was placed in a cryostat evacuated to a base pressure of $1 \times 10^{-3} \mathrm{mbar}$. Good thermal contact between the studied specimens and the metallic heating stage was ensured by using silver paint. The linear dependence of the LO frequency on the temperature was obtained in the range from 293 to $363 \mathrm{~K}$ and is shown in Fig. 5. For an externally induced temperature rise of $70 \mathrm{~K}$ in the microcrystals, a change in the LO frequencies of about $1.2 \mathrm{~cm}^{-1}$ was measured. From the linear fit to the experimental data in Fig. 5, the temperature coefficient of the LO phonon frequency shift was determined as $\mathrm{d} \Delta \omega / \mathrm{d} T=-0.0171 \mathrm{~cm}^{-1} \mathrm{~K}^{-1}$, which is in good agreement with the specific temperature dependence of the LO mode of $\mathrm{d} \Delta \omega / \mathrm{d} T=-0.0168 \mathrm{~cm}^{-1} \mathrm{~K}^{-1}$, reported by Irmer and coworkers $^{32}$ for single-crystalline bulk InP (filled squares in Fig. 5). In addition, we studied a bulk monocrystalline (100) InP substrate to validate the method, which yielded a linear temperature coefficient of $\mathrm{d} \Delta \omega / \mathrm{d} T=-0.0165 \mathrm{~cm}^{-1} \mathrm{~K}^{-1}$, which is also in good agreement with the previously reported value.

\subsection{Numerical simulation}

The thermal conductivity of InP microcrystals was obtained by solving the steady-state heat conduction equation for volu-

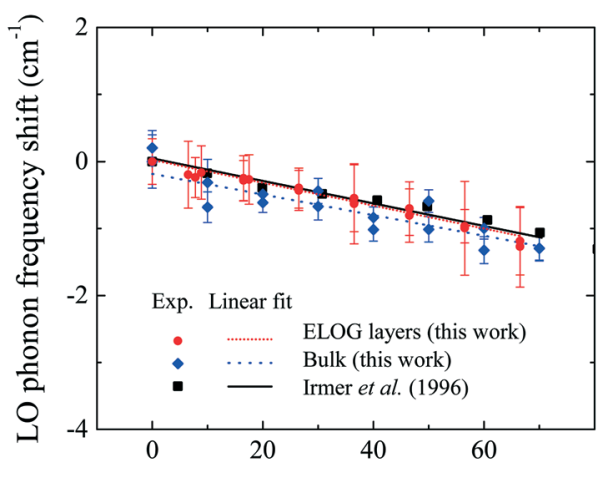

Relative temperature rise $(\mathrm{K})$

Fig. 5 Linear dependence of the $\operatorname{InP}$ LO peak position on temperature. The red dots refer to the microcrystals, the blue diamonds to the bulk sample and the black squares to the work of Irmer et al. ${ }^{32}$ metric Gaussian thermal excitation and comparing it to the experimental data. For micrometric samples, an approximate analytical solution is often employed assuming the specimen as a semi-infinite medium with strong absorption at the surface. ${ }^{33-35}$ Yet, owing to variations in the dimensions of our samples during growth, the microcrystals could not be considered as semi-infinite volumes, particularly when the width of the ELOG InP layers, $b$, was comparable to the laser spot diameter $(2 r \geq b)$. To account for these effects in the temperature distribution, we solved the three-dimensional (3D) stationary problem of a Raman thermometry experiment by numerical analysis based on the FEM. The physical formulation was developed in Cartesian coordinates such that the $x-y$ plane was defined on the surface of the microcrystal and the incident laser beam was oriented along the $z$-direction.

Assuming that the laser excitation has a Gaussian profile, the volumetric thermal source $Q_{0}(x, y)$ can be written according to the Beer-Lambert law:

$$
Q_{0}(x, y)=\frac{2 P_{\mathrm{i}}(1-R)}{\pi r^{2} \rho} \exp \left[-2 \frac{\left(x^{2}+y^{2}\right)}{r^{2}}\right]
$$

and therefore the governing differential equation for heat conduction in the steady-state can be written as:

$$
Q_{0}(x, y)=-\nabla \cdot(\kappa \nabla T)
$$

where $\kappa$ is the thermal conductivity and $T$ is the temperature. Eqn (2) represents a 3D problem that can be conveniently solved by finding an approximate numerical solution with finite element analysis using proper computer software, such as COMSOL®. To do so, the studied specimen was discretized in nodal elements. The boundary conditions at the top and lateral surfaces of the microcrystal were assumed to be adiabatic, while a temperature of $T=293.15 \mathrm{~K}$ was set at the bottom face. This means that convection and radiation toward the surrounding environment were considered to be negligible and the thermal contact between the sample and the holder was assumed to be excellent. It is worth noting that for the doped set of samples, the $600 \mathrm{~nm}$ thick $\mathrm{SiO}_{2}$ mask was included in the modeling by considering an additional rectangular volume in perfect thermal contact with the microcrystal. In this case, the isothermal boundary condition was prescribed at the bottom of the $\mathrm{SiO}_{2}$ layer, and the thermal boundary resistance between InP and $\mathrm{SiO}_{2}$ was considered to be negligible. Since the temperature rise in the experiments did not exceed $100 \mathrm{~K}$, a constant thermal conductivity was assumed. The Gaussian heat source was applied to a cylinder on the top surface, defined as the probed volume (inset to Fig. 6). This domain, which was specified with the dimensions dictated by the laser beam profile, was used to extract a weighted average of the temperature across the probed region. Exploiting the symmetry of the problem, the simulation took place on half of a sample volume. A scheme of the 3D heat diffusion problem, representing the radial heat flux 


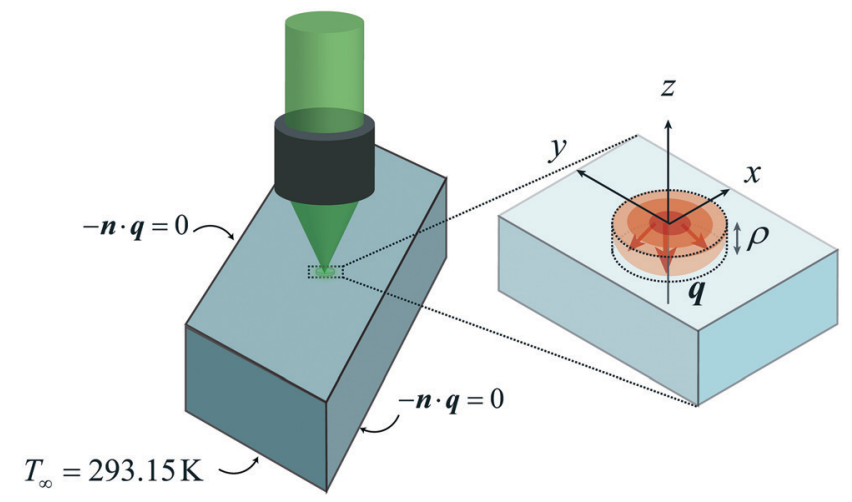

Fig. 6 Schematic illustration of the 3D heat diffusion problem displaying the radial heat flux induced by a Gaussian laser on an ELOG InP microcrystal. The boundary conditions used for the calculations are adiabatic at the top and at the lateral surfaces of the microcrystal $(-\boldsymbol{n} \cdot \boldsymbol{q}=0$, where $\boldsymbol{n}$ is the unit vector normal to the surface and $\boldsymbol{q}$ is the heat flux) and isothermal at the bottom of the sample $\left(T_{\infty}=293.15 \mathrm{~K}\right)$. The inset shows the cylindrical domain where the volumetric Gaussian heat source was applied.

induced by a Gaussian laser on an ELOG microcrystal, is presented in Fig. 6.

The computation of the boundary fluxes involved solving a system of linear equations derived for each volume element. By using the superposition principle, the temperature field was obtained from these nodal heat fluxes. Knowing the exact dimensions of the InP crystals is of crucial importance for the accurate calculation of the temperature profile via numerical methods. Therefore, the lateral dimensions of each microcrystal were measured by scanning electron microscopy (ZEISS GEMINI ULTRA 55), and the thickness was obtained by optical profilometry (Veeco Wyko NT9300). The measured geometry parameters were used as individual geometrical inputs for the numerical model.

\section{Results and discussion}

\subsection{InP microcrystals: morphology and crystalline quality}

Raman scattering provides a very quick and simple way to determinate the crystalline quality of the material. Typically, the Raman signal is very sensitive to strain, ${ }^{36}$ sample composition, doping, structural defects, ${ }^{37}$ crystallographic orientations, ${ }^{38}$ and pseudo-phases, ${ }^{39}$ among others. According to Raman selection rules applicable to III-V semiconductors with a zinc blende structure, the TO phonon mode is symmetryforbidden in (100)-oriented surfaces. In this case, only a sharp and intense LO peak can be observed in the Raman measurements obtained in the backscattering geometry. In general, this peak is used as an indication of the long-range order of the crystal. However, structural disorder, impurities and alloying can break the translational symmetry and activate the TO mode. ${ }^{40}$ Then, the intensity ratio of the two peaks (LO/TO or TO/LO) provides an indication of the structural defects. Fig. 7 illustrates this, where the intensity ratios of LO/TO (Fig. 7a) and TO/LO (Fig. 7b) of an undoped micro- crystal grown through a $300 \mathrm{~nm}$ trench are depicted as examples. The high quality of the ELOG InP crystal is demonstrated with the high intensity of the LO/TO ratio, as shown in Fig. 7a. In contrast, the InP seed layer, which is highly defective, is shown to be less intense in the LO/TO ratio but rather intense in the $\mathrm{TO} / \mathrm{LO}$ ratio, as displayed in Fig. 7b. An estimate of the intensity was obtained by means of a Lorentzian fit of each spectrum with a mean error of $\sim 10 \%$ and $0.03 \%$ for TO and LO fitted intensities, respectively. Fig. 7c is a schematic illustration of the specimen cross section and Fig. $7 \mathrm{~d}$ is a Raman spectrum obtained at $P_{\mathrm{i}}=6 \mathrm{~mW}$, showing the TO and LO peaks for the analyzed sample. Further characterization showing the high quality of the InP crystals, including high resolution X-ray diffraction data and AFM measurements, can be found in the ESI. $\dagger$

Scanning electron microscopy and optical profilometry were used for measuring the dimensions and characterizing the morphology of the grown InP microcrystals. These analyses showed that the trench width strongly affects the crystal growth and defines the dimensions of the microcrystals. From Fig. 8 it can be observed that the crystal thickness, width and volume increase with increasing trench width for both sets of samples. The results also show that doped microcrystals are systematically smaller (Fig. 8e and g) and exhibit slightly different morphologies (Fig. 8c and d) from their undoped counterparts (Fig. 8a and b).

\subsection{Thermal properties}

The applicability of the Raman thermometry method to the ELOG layers was first validated on a sample of a bulk monocrystalline (100) InP substrate. The dependence of the relative Raman shift on the power absorbed at the surface of the InP substrate and microcrystals is presented in Fig. 9. Using the calibration curves in Fig. 5, the local temperature rise for each absorbed power can be calculated considering that:

$$
\Delta T=\omega-\omega_{0}\left(\frac{\mathrm{d} \Delta \omega}{\mathrm{d} T}\right)^{-1}
$$

where $\omega$ and $\omega_{0}$ are the measured Raman shift and the frequency of the studied phonon mode at room temperature, respectively. From the series of measurements on the InP bulk substrate where the excitation power is gradually increased, the LO peak redshift with absorbed power was estimated according to $\mathrm{d} \Delta \omega / \mathrm{d} P=-0.0383 \mathrm{~cm}^{-1} \mathrm{~mW}^{-1}$ (open hexagons in Fig. 9). Using the linear dependence of $\mathrm{d} \Delta \omega / \mathrm{d} T=-0.0165$ $\mathrm{cm}^{-1} \mathrm{~K}^{-1}$ obtained from the calibration procedure (Fig. 5) and fitting the experimental temperature rise to the $3 \mathrm{D}$ model, a thermal conductivity of $\kappa_{\text {bulk }}=69.6 \mathrm{~W} \mathrm{~m}^{-1} \mathrm{~K}^{-1}$ was calculated. With a slight overestimation of about $2 \%$, this result is in fair agreement with the accepted value of bulk InP thermal conductivity of $\kappa_{\mathrm{InP}}=68 \mathrm{~W} \mathrm{~m}^{-1} \mathrm{~K}^{-1}$ at room temperature, ${ }^{41}$ and therefore validates the numerical method.

A clear trend for both undoped and doped samples emerges from the results presented in Fig. 9. The temperature 
a)

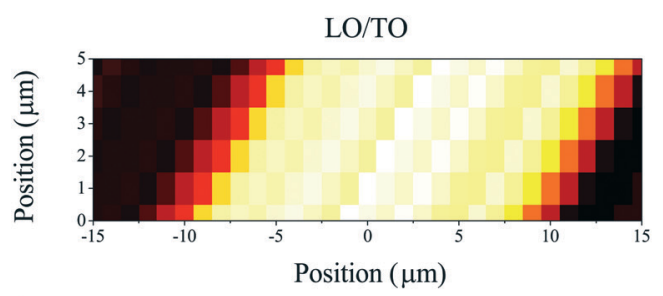

b)

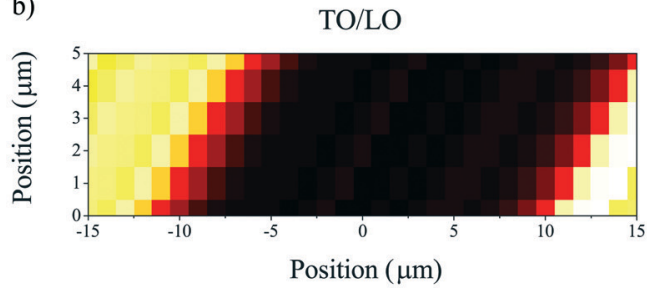

c)

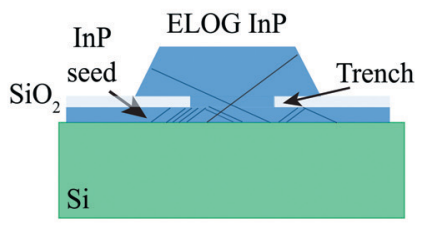

d)

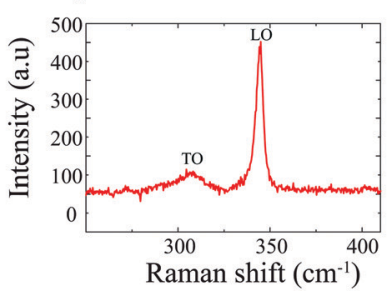

Fig. 7 Raman spectral mapping of the investigated InP/Si sample. a) Normalized intensity ratio of LO to TO modes for an undoped ELOG microcrystal (center) and the InP seed layer underneath (sides). b) Intensity ratio of TO/LO modes for the same specimen. c) Schematic illustration of the cross section. d) Raman spectrum of the analyzed sample, exhibiting the TO and LO peaks.
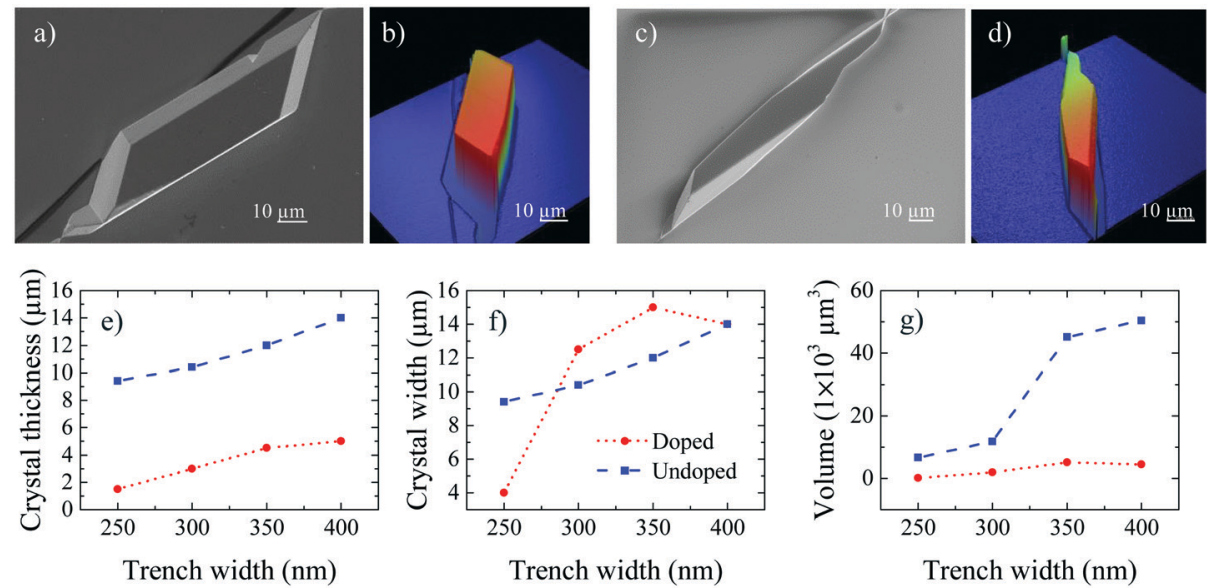

Fig. 8 Scanning electron micrographs and optical profilometry scans: a) and b) for undoped and c) and d) for doped ELOG InP microcrystals, respectively. The analyzed samples were grown through trenches of $300 \mathrm{~nm}$ width. The red color of the color scales in b) and d) corresponds to the maximum thickness values of 10.4 and $3 \mu \mathrm{m}$ for the undoped and doped microcrystals, respectively, while the scale bars of $10 \mu \mathrm{m}$ refer to the lateral dimensions. e)-g) are the measured crystal thickness, width and volume of doped (red circles) and undoped (blue squares) samples as a function of the trench width.

rise upon light absorption is considerably larger for the microcrystals grown from smaller trenches. This observation can be understood as the result of the reduced dimensions of the crystals grown through narrower trenches, which ultimately leads to smaller effective volumes that can dissipate the heat and thus reach higher temperatures. Regarding the undoped samples, the microcrystals grown through 400 and $350 \mathrm{~nm}$ wide trenches have similar temperature rises as the bulk sample, while the ones grown through $250 \mathrm{~nm}$ and 300 $\mathrm{nm}$ wide trenches show slightly higher temperature rises. Moreover, the doped samples are heated to higher temperatures compared to the bulk substrate and their undoped counterparts. This can be explained by two factors: the small dimensions of the doped crystals and the low thermal conductivity of the $600 \mathrm{~nm}$ thick $\mathrm{SiO}_{2}$ layer $\left(\kappa_{\mathrm{SiO}_{2}}=1.4 \mathrm{~W} \mathrm{~m}{ }^{-1} \mathrm{~K}^{-1}\right.$ (ref. 42)), which promote the heating of the microcrystals.
The experimental values of thermal conductivity measured in the bulk InP substrate, undoped and doped ELOG microcrystals are presented in Fig. 10. These were extracted from the power absorbed at the sample surface, the resulting temperature rise and the solution to the heat diffusion equation solved by the finite element method. Thermal conductivities of $68 \pm 4$ and $74 \pm 8 \mathrm{~W} \mathrm{~m}^{-1} \mathrm{~K}^{-1}$ were obtained for undoped microcrystals grown through trenches of 400 and $350 \mathrm{~nm}$, respectively. The doped sample grown through a $400 \mathrm{~nm}$ wide trench also has a thermal conductivity of $68 \pm 6 \mathrm{~W} \mathrm{~m}^{-1} \mathrm{~K}^{-1}$. These results, which are very close to the bulk value, constitute evidence of the high crystalline quality of the monocrystalline ELOG samples grown through wider trenches and are in agreement with the temperature rises reported in Fig. 9. The slight overestimation of about $8 \%$ for the undoped sample grown though a $350 \mathrm{~nm}$ wide trench is within the experimental error, and might be due 


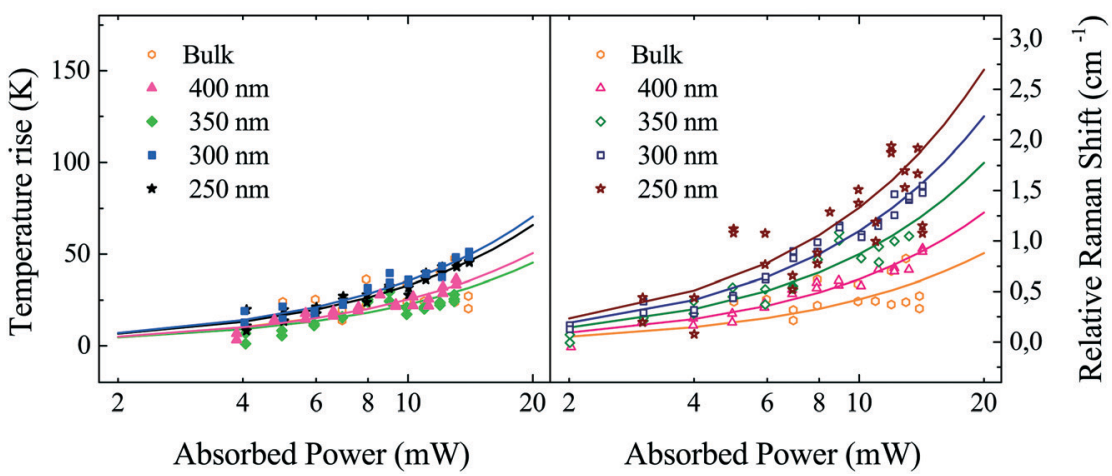

Fig. 9 Relative Raman shift and corresponding temperature rise as a function of the power absorbed, measured for the studied InP bulk substrate, and a) the undoped as well as b) the doped microcrystals.

to the propagation of uncertainties related to the experimental determination of the laser spot size, the optical absorption and sample dimensions.

The results in Fig. 10 also suggest that the thermal conductivity of the microcrystals grown through narrower trenches is below the bulk value. For the undoped samples, the thermal conductivity decreased by 31 and $29 \%$ as the trench width was reduced to 250 and $300 \mathrm{~nm}$, respectively. Similarly, the thermal conductivity of the doped samples grown though equivalent trench widths showed a decrease of about $54 \%$ with respect to the bulk value. On the other hand, the value of the thermal conductivity of the doped sample grown through a $350 \mathrm{~nm}$ wide trench implies a reduction from the bulk value of about $38 \%$. These results might seem counterintuitive because it is expected that narrower trenches filter stacking faults and threading dislocations more effectively than the wider ones. However, this depends on the aspect ratio. Junesand and co-workers showed that stacking faults are completely blocked by the $\mathrm{SiO}_{2}$ mask when the aspect ratio of the mask height to the opening width is equal to

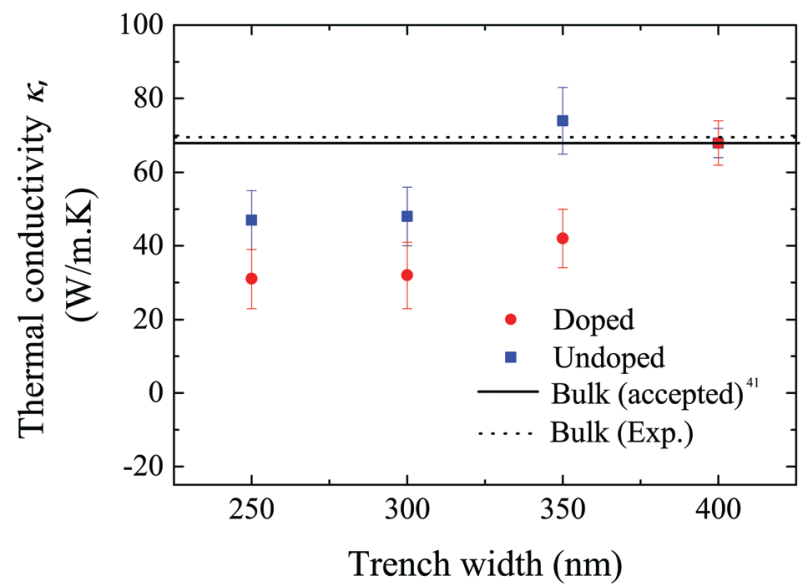

Fig. 10 Thermal conductivity of undoped and doped ELOG InP microcrystals as a function of the trench width through which they are grown. The dashed line indicates the measurement of the bulk InP thermal conductivity. The solid line represents the generally accepted value of thermal conductivity for bulk InP. ${ }^{41}$ or larger than $3.9 .^{26}$ The samples studied here have a maximum aspect ratio of about 2.4 , and thus complete dislocation filtering in the samples reported here is disregarded. Transmission electron microscopy studies reported elsewhere ${ }^{26,27}$ have shown that stacking fault propagation takes place even for narrower trench widths in both undoped and doped samples. In addition, these studies showed that stacking faults do not always reach the surface of the ELOG layers, but appear to be filtered at some point close to the mask opening in the direction perpendicular to the Si substrate, depending on the mask aspect ratio. In a separate study of AlN films on a Si substrate, we have demonstrated ${ }^{43-46}$ that the contribution to the effective thermal conductivity by phonon scattering at crystallographic defects becomes stronger as the sample thickness decreases. This means that the thermal conductivity is greatly reduced for smaller feature films because phonon scattering at the interface and crystallographic defects dominates thermal transport. Thus, the reduction of the thermal conductivity measured in InP microcrystals grown through narrower trenches (Fig. 10) can be associated with phonon scattering at stacking faults close to the interface between the InP ELOG layer and the $\mathrm{SiO}_{2}$ mask as the dimensions of the microcrystals decrease. On the other hand, the ELOG layers grown though wider openings are much thicker, and the stacking faults are filtered in the region close to the interface with the $\mathrm{SiO}_{2}$ masks. Therefore, the phonon scattering at the interface and crystallographic defects is no longer predominant, but thermal transport is dominated by phonon-phonon scattering, as in bulk single crystalline InP. This observation is supported by the bulk values measured for the thermal conductivities of thicker microcrystals, which are grown through wider trenches, i.e., undoped samples grown through openings of 350 and $400 \mathrm{~nm}$ and the doped specimen grown through the $400 \mathrm{~nm}$ trench. Based on these results, we propose that thicker and wider ELOG layers, where defects do not reach the surface but are filtered in a region close to the mask opening, are better suited for integration of active devices such as laser sources. The two main advantages are the high thermal conductivity of ELOG InP microcrystals close to the bulk value and a larger effective 
volume through which heat can be dissipated. On the other hand, thinner ELOG layers in close contact with the $\mathrm{SiO}_{2}$ mask result in much higher temperature rises and therefore are less efficient in removing heat.

\section{Conclusions}

The thermal conductivity of ELOG InP on silicon has been experimentally investigated via microRaman thermometry. In this work, both undoped and sulfur-doped samples grown through trenches of widths from 250 to $400 \mathrm{~nm}$ were studied. The impact of the ELOG layer size and crystallographic defects on thermal transport was analyzed. Our results show that the thermal conductivity of doped and undoped ELOG InP on silicon is very close to the bulk value for samples grown through trenches of $400 \mathrm{~nm}$ width. We attribute this to the fact that the samples are thicker and have a lower defect density, consistent with previous work on dislocation filtering. In this case, the thermal transport is dominated by phonon-phonon interactions as in a monocrystalline bulk material. Based on these results, we propose that thicker ELOG microcrystals are better suited for applications involving light source integration due to the higher thermal conductivity and a larger effective volume through which the generated heat is dissipated. The trend and reduction of the thermal conductivity may help to improve thermal analyses in a variety of hybrid integrated opto-electronic circuits.

\section{Acknowledgements}

This research was supported by the Swedish Research Council VR (grant numbers 349-2007-8664 and 2014-5100) and the Linnaeus Center in Advanced Optics and Photonics (ADOPT). CMST and ECA gratefully acknowledge support from the Spanish MINECO (projects PHENTOM FIS2015-70862-P and nanoTHERM CSD2010-00044), the Catalan AGAUR and the MINECO Severo Ochoa program grant SEV-2013-0295. JJF would like to thank especially Dr. M. Sledzinska and Dr. B Graczykowski for helpful discussions and assistance with the Raman thermometry measurements.

\section{References}

1 J. E. Bowers, T. Komljenovic, M. Davenport, J. Hulme, A. Y. Liu, C. T. Santis, A. Spott, S. Srinivasan, E. J. Stanton and C. Zhang, Proceedings of SPIE, Next-Generation Optical Communication: Components, Sub-Systems, and Systems V, 2016, vol. 9774, pp. 1-18.

2 P. Reithmaier and M. Benyoucefa, ECS Trans., 2016, 72, 171-179.

3 W. Metaferia, A. Dev, H. Kataria, C. Junesand, Y.-T. Sun, S. Anand, J. Tommila, G. Pozina, L. Hultman, M. Guina, T. Niemi and S. Lourdudoss, CrystEngComm, 2014, 16, 4624-4632.

4 Y.-T. Sun, H. Kataria, W. Metaferia and S. Lourdudoss, CrystEngComm, 2014, 16, 7889-7893.

5 D. Liang, X. Huang, G. Kurczveil, M. Fiorentino and R. G. Beausoleil, Nat. Photonics, 2016, 10, 1-4.
6 J. Michel, J. Liu and L. C. Kimerling, Nat. Photonics, 2010, 4, 527-534.

7 G. T. Reed, G. Mashanovich, F. Y. Gardes and D. J. Thomson, Nat. Photonics, 2010, 4, 518-526.

8 J. Liu and R. Yang, Phys. Rev. B: Condens. Matter Mater. Phys., 2010, 81, 174122.

9 S. Chen, W. Li, J. Wu, Q. Jiang, M. Tang, S. Shutts, S. N. Elliott, A. Sobiesierski, A. J. Seeds, I. Ross, P. M. Smowton and H. Liu, Nat. Photonics, 2016, 10, 307-312.

10 Y. Mols, B. Kunert, G. Gaudin, R. Langer and M. Caymax, J. Cryst. Growth, 2016, 452, 244-247.

11 F. Gao, L. Wen, X. Zhang, Y. Guan, J. Li, S. Zhang and G. Li, Thin Solid Films, 2015, 589, 32-37.

12 F. Gao, L. Wen, Y. Guan, J. Li, X. Zhang, M. Jia, S. Zhang and G. Li, CrystEngComm, 2014, 16, 10721-10727.

13 H. A. Fonseka, H. H. Tan, J. Wong-Leung, J. H. Kang, P. Parkinson and C. Jagadish, Nanotechnology, 2013, 24, 465602.

14 C. K. Majumdar, J. Math. Phys., 1969, 10, 1388.

15 A. Iskandar, A. Abou-Khalil, M. Kazan, W. Kassem and S. Volz, J. Appl. Phys., 2015, 117, 125102.

16 M. N. Sysak, D. Liang, R. Jones, G. Kurczveil, S. Member, M. Piels, S. Member, M. Fiorentino, R. G. Beausoleil, S. Member and J. E. Bowers, IEEE J. Sel. Top. Quantum Electron., 2011, 17, 1490-1498.

17 S. Lei, I. Mathews, J. Camus, S. Bensalem, M. A. Djouadi, A. Shen, G.-H. Duan and R. Enright, 2016 15th IEEE Intersociety Conference on Thermal and Thermomechanical Phenomena in Electronic Systems (ITherm), 2016, vol. 1, pp. 1024-1029.

18 I. Mathews, S. Lei, K. Nolan, G. Levaufre, A. Shen, B. Corbett and R. Enright, Proceedings of SPIE, Integrated Photonics: Materials, Devices, and Applications III, 2015, vol. 9520, pp. 1-10.

19 I. Lucci, C. Cornet, M. Bahri and Y. Léger, IEEE J. Sel. Top. Quantum Electron., 2016, PP, 1-9.

20 D. G. Cahill, W. K. Ford, K. E. Goodson, G. D. Mahan, A. Majumdar, H. J. Maris, R. Merlin and S. R. Phillpot, J. Appl. Phys., 2003, 93, 793.

21 Z. Zhou, B. Yin and J. Michel, Light: Sci. Appl., 2015, 4, e358.

22 S. Lourdudoss, Curr. Opin. Solid State Mater. Sci., 2012, 16, 91-99.

23 Y. B. Bolkhovityanov and O. P. Pchelyakov, Open Nanosci. J., 2009, 3, 20-33.

24 Z. Wang, C. Junesand, W. Metaferia, C. Hu, L. Wosinski and S. Lourdudoss, Mater. Sci. Eng., B, 2012, 117, 1551-1557.

25 T. A. Langdo, C. W. Leitz, M. T. Currie, E. A. Fitzgerald, A. Lochtefeld and D. A. Antoniadis, Appl. Phys. Lett., 2000, 76, 3700-3702.

26 C. Junesand, H. Kataria, W. Metaferia, N. Julian, Y.-T. Sun, J. Bowers, G. Pozina and L. Hultman, Opt. Mater. Express, 2013, 3, 1960-1973.

27 H. Kataria, W. Metaferia, C. Junesand, C. Zhang, N. Julian and J. E. Bowers, IEEE J. Sel. Top. Quantum Electron., 2014, 20, 380-386.

28 G. Lucazeau, J. Raman Spectrosc., 2003, 4, 478-496.

29 T. E. Beechem and J. R. Serrano, Spectroscopy, 2011, 26, 36-44. 
30 D. E. Aspnes and A. A. Studna, Phys. Rev. B: Condens. Matter Mater. Phys., 1983, 27, 985-1009.

31 D. J. Lockwood, G. Yu and N. L. Rowell, Solid State Commun., 2005, 136, 404-409.

32 G. Irmer, M. Wenzel and J. Monecke, Phys. Status Solidi B, 1996, 195, 85-95.

33 B. Stoib, S. Filser, J. Stötzel, A. Greppmair, N. Petermann, H. Wiggers, G. Schierning, M. Stutzmann and M. S. Brandt, Semicond. Sci. Technol., 2014, 29, 124005.

34 S. Perichon, V. Lysenko, B. Remaki, D. Barbier and B. Champagnon, J. Appl. Phys., 1999, 86, 4700.

35 V. Lysenko, S. Perichon, B. Remaki, D. Barbier and B. Champagnon, J. Appl. Phys., 1999, 86, 6841-6846.

36 Y. Ogawa, T. Toizumi, F. Minami and A. V. Baranov, Phys. Rev. B: Condens. Matter Mater. Phys., 2011, 83, 081302.

37 S. Fuentes, P. Muñoz, P. Barraza, E. Chavez-Angel and C. M. Sotomayor Torres, J. Sol-Gel Sci. Technol., 2015, 75, 593-601.
38 T. Muck, W. Wagner, L. Hansen, V. Wagner and J. Geurts, J. Appl. Phys., 2001, 95, 54035407.

39 E. Chávez-Angel, S. Fuentes, R. Zarate and L. PadillaCampos, J. Mol. Struct., 2010, 984, 131-136.

40 R. Cuscó, G. Talamàs, L. Artús, J. M. Martin and G. González-Díaz, J. Appl. Phys., 1996, 79, 3927-3929.

41 P. D. Maycock, Solid-State Electron., 1967, 10, 161-168.

42 S.-M. Lee and D. G. Cahill, J. Appl. Phys., 1997, 81, 2590-2595.

43 J. Jaramillo-Fernandez, J. Ordonez-Miranda, E. Ollier and S. Volz, Phys. Chem. Chem. Phys., 2015, 17, 8125-8137.

44 J. Jaramillo-Fernandez, $P h D$ thesis, Ecole Centrale Paris, 2015.

45 J. Jaramillo-Fernandez, J. Ordonez-Miranda, W. Kassem, E. Oilier and S. Volz, 2015 21st International Workshop on Thermal Investigations of ICs and Systems (THERMINIC), 2015, pp. 1-6.

46 J. Jaramillo-Fernandez, J. Ordonez-Miranda, E. Ollier, R. Sanatinia, H. Kataria, E. Chavez-Angel, S. Volz and C. M. Sotomayor Torres, ECS Trans., 2015, 69, 53-64. 